\section{Epithelial Dystrophies}

Saeed Alwadani

Department of Ophthalmology, King Saud

University, Riyadh, Kingdom of Saudi Arabia

\section{Synonyms}

Anterior corneal dystrophies; Epithelial basement membrane dystrophy (EBMD); Lisch epithelial corneal dystrophy; Meesman's epithelial dystrophy

\section{Definition}

Epithelial dystrophy is a bilateral disease that affects the outermost layer of the cornea and is characterized by intraepithelial cysts and abnormal basement membrane, leading to recurrent epithelial erosions, which can be painful and cause blurred vision. There are three subtypes of epithelial dystrophy including EBMD which is the most common anterior corneal dystrophy seen by the comprehensive ophthalmologist, Meesman's epithelial dystrophy, and the Lisch epithelial corneal dystrophy. EBMD is often sporadic but may be inherited in an autosomal dominant fashion; point mutations in the TGFB1 gene have been associated in a minority of cases.

\section{Further Reading}

Eagle RC. Eye pathology: an atlas and text, 2nd edn. Wolters Kluwer, Philadelphia

Weisenthal R (2013-2014a) Section 8: external disease and cornea. In: American Academy of Ophthalmology (ed) Basic and Clinical Science Course (BCSC). American Academy of Ophthalmology, San Francisco

Weisenthal R (2013-2014b) Section 4: ophthalmic pathology and intraocular tumors. In: American Academy of Ophthalmology (ed) Basic and Clinical Science Course (BCSC). American Academy of Ophthalmology, San Francisco 